\title{
Continuous and Discrete Representations of an Iterative Reproduction Method
}

\author{
JÁNOS IMHOF
}

Aluterv-Fki, Budapest $\dagger$

(Received 30 January 1987, in final form 10 April 1987)

Dedicated to the memory of Professor Günter Wassermann

General formulae are obtained for iterative reproduction of the distribution of orientations from both unreduced and reduced pole-figures. The most correct representation of them is the continuous one realized by the Bunge-Roe formalism. The discrete representations are always connected with the additional assumption that the texture function is considered to be constant inside the subsets of orientation space. One of the discrete representations contains the elements of the elementary pole-figures of the vector method.

KEY WORDS: Continuous representations, discrete representations, Bunge-Roe formalism, pole-figures.

\section{INTRODUCTION}

We summarize and explain the designations we shall use in this work.

$K_{A}$ : right-handed orthogonal coordinate system fixed to the sample.

$\vec{y}_{k}: \quad$ sample vector with serial number $k(k=1, \ldots, K)$.

$K_{B}$ : right-handed orthogonal coordinate system fixed to the crystallite according to a given prescription, taking into account the crystal structure of the crystallite.

† H-8005 Székesfehérvár, Pf. 10, Hungary. 


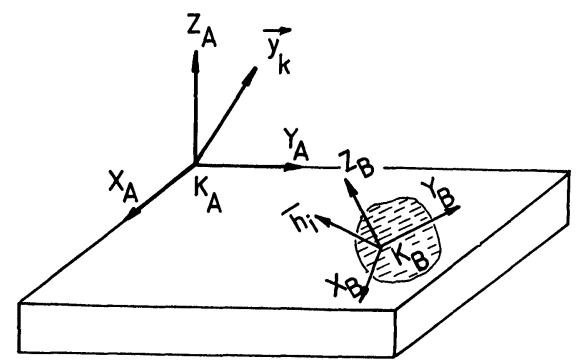

Figure 1 Sample coordinate system $K_{A}\left\{X_{A}, Y_{A}, Z_{A}\right\}$ and crystal coordinate system $K_{B}\left\{X_{B}, Y_{B}, Z_{B}\right\}$. Sample vector $\vec{y}_{k}$ and crystal vector $\vec{h}_{i}$.

$\vec{h}_{i}$ : physically distinguishable crystal vectors $(i=1, \ldots, I)$, Figure 1.

$\mathscr{G}_{B}: \quad$ point symmetry group of the crystallites.

$g_{b_{e}}: \quad$ elements of the point symmetry group $\mathscr{G}_{B}$

$$
g_{b_{e}} \in \mathscr{G}_{B}, \quad e=1, \ldots, N_{b} .
$$

$K_{B_{e}}: \quad$ orthogonal coordinate systems equivalent to $K_{B}$

$$
K_{B_{e}}=g_{b_{e}}^{p} \cdot K_{B}, \quad e=1, \ldots, N_{b} .
$$

Here we use the upperscript $p$ to emphasize that we mean a passive operator. A passive operator is one that moves the axes of space, all points of the space and hence all vector positions being left unmoved, so that after each operation a vector position is referred to a new set of axes (Bradley and Cracknell, 1972).

$u_{e}$ : orthogonal transformation which transforms $K_{A}$ into $K_{B_{e}}$ (Bunge, Esling and Müller, 1982)

$$
K_{B_{e}}=u_{e} \cdot K_{A}, \quad e=1, \ldots, N_{b} .
$$

Generally, the set $\{u\}$ of all possible orthogonal transformations consists of two separated subsets, $G$ and $U$, containing only proper and improper rotations, respectively. From any proper rotation $g \in G$ the equivalent orthogonal transformations can be got by the equation

$$
u_{e}=g_{b_{e}}^{p} \cdot g, \quad e=1, \ldots, N_{b} .
$$

The matrix elements describing the proper rotation $g$ are 
usually expressed by the Eulerian angles $\varphi_{1}, \Phi, \varphi_{2}$ (Bunge, 1969) or $\alpha, \beta, \gamma$ (Matthies, 1982) in the region

$$
G: 0 \leqslant \alpha<2 \pi, \quad 0 \leqslant \beta \leqslant \pi, \quad 0 \leqslant \gamma<2 \pi .
$$

$f(g)$ : orientation density function describing the distribution of proper rotations. The definition is

$$
\frac{\Delta V}{V}\left(g \in G_{j}\right)=\frac{1}{\int_{G} d g} \int_{G_{j}} f(g) d g
$$

where $G_{j}$ : arbitrary subset of the region $G(j=$ $1, \ldots, \mathscr{F}), \Delta V / V\left(g \in G_{j}\right)$ : relative volume of all the crystallites with $g \in G_{j}$.

$\left\{\vec{h}_{i}\right\}$ : complete set of all the crystal vectors equivalent to $\vec{h}_{i}$

$$
\left\{\vec{h}_{i}\right\}=\left\{\vec{h}_{i_{1}}, \vec{h}_{i_{2}}, \ldots, \vec{h}_{i_{N_{b}}}\right\}
$$

where

$$
\vec{h}_{i_{e}}=g_{b_{e}}^{a} \cdot \vec{h}_{i}, \quad e=1, \ldots, N_{b} .
$$

Here we use the upperscript $a$ to emphasize that we mean an active operator. An active operator is one that moves the points or position vectors of space, all vectors being referred to a fixed set of axes (Bradley and Cracknell, 1972).

Example. Let $\mathscr{G}_{B}=C_{2 v}$. The elements $g_{b_{e}}$ given in the set of axes $K_{B}$ are: $g_{b_{1}}=E$ (identity), $g_{b_{2}}=C_{2 z}, g_{b_{3}}=\sigma_{x}$, $g_{b_{4}}=\sigma_{y} \cdot \vec{h}_{i}$ is an arbitrarily chosen crystal vector. By Eq. (8) we get $N_{b}=4$ vectors $\vec{h}_{i_{e}}$ equivalent to $\vec{h}_{i}$, Figure 2 .

$T_{i_{e} k}$ : the set of all particular orientations in the region $G(5)$ at which the vector $\vec{h}_{i_{e}}$ described in $K_{B}$ is parallel to a fixed sample vector $\vec{y}_{k}$. The subscripts of $T_{i_{e} k}$ refer to that of $\vec{h}_{i_{e}}$ and $\vec{y}_{k}$, respectively. Example. Let us consider a single crystal with $\mathscr{G}_{B}=C_{2 v}$ and its vector set $\left\{\vec{h}_{i}\right\}$, Figure 2. $\vec{y}_{k}$ is an arbitrarily chosen sample vector. We rotate the single crystal till $\vec{h}_{i_{1}}$ is parallel with $\vec{y}_{k}$. At this moment let the orientation of $K_{B}$ with respect to $K_{A}$ be $g_{1}$. After rotating the crystal around $\vec{h}_{i_{1}}$ we get all orientations in $G$ at which $\vec{h}_{i_{1}}$ is parallel to $\vec{y}_{k}$ i.e., the set $T_{i_{1} k}$. Any set $T_{i_{e} k}$ can be constructed in the same way.

The set $T_{i_{e} k}$ can mathematically be described as follows. The coordinates of $\vec{y}_{k}=\left(X_{k}, Y_{k}, Z_{k}\right)$ are given in $K_{A}$, while 

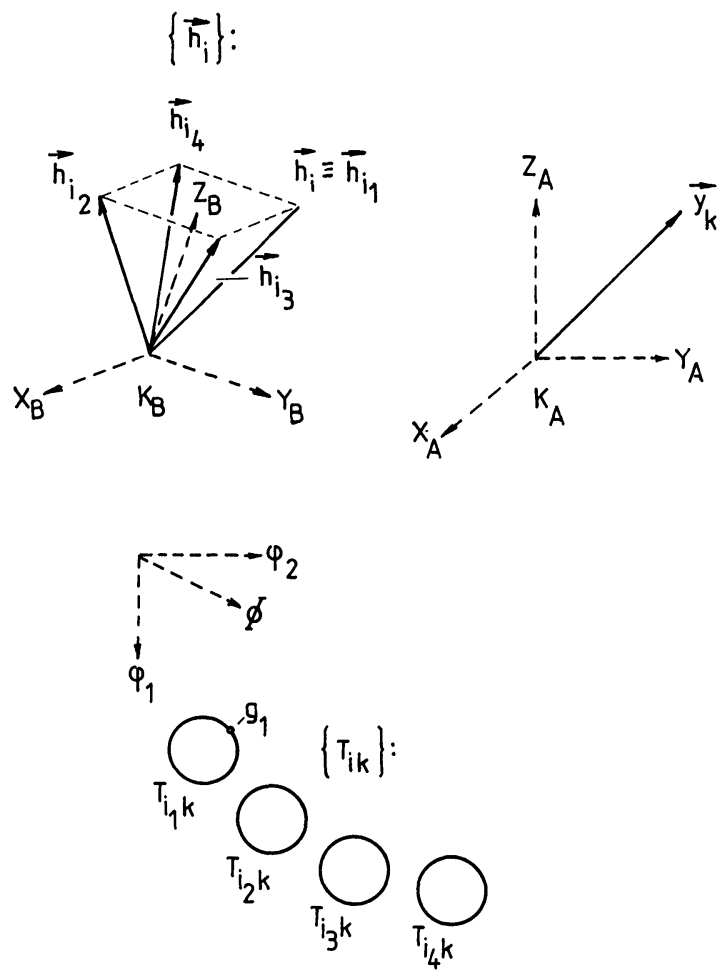

Figure 2 Example for the sets $\left\{h_{i}\right\}, T_{i_{e} k}$ and $\left\{T_{i k}\right\}$ in the case of $\mathscr{G}_{B}=C_{2 v} . \vec{h}_{i}$ is an arbitrarily chosen crystal vector. By Eq. (8) we get $N_{b}=4$ vectors $h i_{e}$ equivalent to $\vec{h}_{i} . \vec{y}_{k}$ is an arbitrary sample vector. Let $g_{1}$ be an orientation of $K_{B}$ at which $\vec{h}_{i_{1}}$ is parallel to $\vec{y}_{k}$. After rotating $K_{B}$ around $\vec{h}_{i_{1}}$ we get the set $T_{i_{1} k}$. The sum of the sets $T_{i_{e} k}(e=1,2,3,4)$ is denoted by $\left\{T_{i k}\right\}$.

that of $\vec{h}_{i_{e}}=\left(X_{i_{e}}, Y_{i_{e}}, Z_{i_{e}}\right)$ are given in $K_{B}$. All possible Eulerian angles are to be found by which the matrix $[g(\alpha, \beta, \gamma)]$ fulfills the equation

$$
\left(\begin{array}{c}
X_{i_{e}} \\
Y_{i_{e}} \\
Z_{i_{e}}
\end{array}\right)=[g(\alpha, \beta, \gamma)]\left(\begin{array}{c}
X_{k} \\
Y_{k} \\
Z_{k}
\end{array}\right) .
$$

Details about this problem can be found e.g. in the works of Pospiech (1980) and Matthies (1982). 
$\left\{T_{i k}\right\}$ : the set of all orientations in $G(5)$ at which one of the vectors $\vec{h}_{i_{e}}$ is parallel to $\vec{y}_{k}$. Thus, $\left\{T_{i k}\right\}$ is the union of the $N_{b}$ sets $T_{i_{e} k}$, Figure 2 .

$$
\left\{T_{i k}\right\}=T_{i_{1} k} \cup T_{i_{2} k} \cup \ldots \cup T_{i_{N_{b}}} \cdot
$$

What is the set $\left\{T_{i k}\right\}$ needed for? The vectors of the set $\left\{\vec{h}_{i}\right\}$ are physically indistinguishable. After detecting an event $\vec{h}_{i_{e}} \| \vec{y}_{k}$ for the corresponding crystallite orientation $g$ we can only say that $g \in\left\{T_{i k}\right\}$.

$\tilde{\mathscr{G}}_{B}: \quad$ reduced point symmetry group constructed from $\mathscr{G}_{B}$ and the inversion operator $C_{i}$ as follows

$$
\tilde{\mathscr{G}}_{B}=\mathscr{G}_{B} \times C_{i} \text {. }
$$

$g_{b_{\bar{e}}}: \quad$ elements of the reduced point group $\tilde{\mathscr{G}}_{B}$

$$
g_{b_{\tilde{e}}} \in \tilde{G}_{B}, \quad \tilde{e}=1, \ldots, \tilde{N}_{b} .
$$

$\left\{\tilde{h}_{i}\right\}: \quad\left\{\vec{h}_{i_{1}}, \vec{h}_{i_{2}}, \ldots, \vec{h}_{i_{\tilde{N}_{b}}}\right\}$

where

$$
\vec{h}_{i_{\bar{e}}}=g_{b_{\tilde{e}}}^{a} \cdot \vec{h}_{i}, \quad \tilde{e}=1, \ldots, \tilde{N}_{b} .
$$

Figure 3 illustrates the set $\left\{\vec{h}_{i}\right\}=\left\{\vec{h}_{i}\right\} \times C_{i}$ in the case of $\mathscr{G}_{B}=C_{2 v}$. If $\mathscr{G}_{B}$ contains the inversion explicitly, i.e., if the crystallites are centrosymmetrical ones, then $\tilde{\mathscr{G}}_{B}=\mathscr{G}_{B}$, $\tilde{N}_{b}=N_{b}$ and $\left\{\tilde{h}_{i}\right\}=\left\{\vec{h}_{i}\right\}$.

$T_{i_{\bar{e}} k}$ : the set of all orientations in $G(5)$ at which the vector $\vec{h}_{i_{\bar{e}}}$ is parallel to a fixed $\vec{y}_{k}$. Figure 3 illustrates the sets $T_{i_{e} k}$ in the case of $\mathscr{G}_{B}=C_{2 v}$.

$\left\{\tilde{T}_{i k}\right\}$ : the set of all orientations in $G(5)$ at which one of the vectors $\vec{h}_{i_{\bar{e}}}$ is parallel to $\vec{y}_{k}$. Thus, $\left\{\tilde{T}_{i k}\right\}$ is the union of the $\tilde{N}_{b}$ sets $T_{i_{e} k}$, Figure 3 . The construction of the set $\left\{\tilde{T}_{i k}\right\}$ was needed because for a type of measurings (for normal scattering) the vectors of the set $\left\{\vec{h}_{i}\right\}$ are indistinguishable. After detecting an event $\vec{h}_{i_{\bar{e}}} \| \vec{y}_{k}$ by normal scattering, for the corresponding crystallite orientation $g$ we can only say that $g \in\left\{\tilde{T}_{i k}\right\}$. 


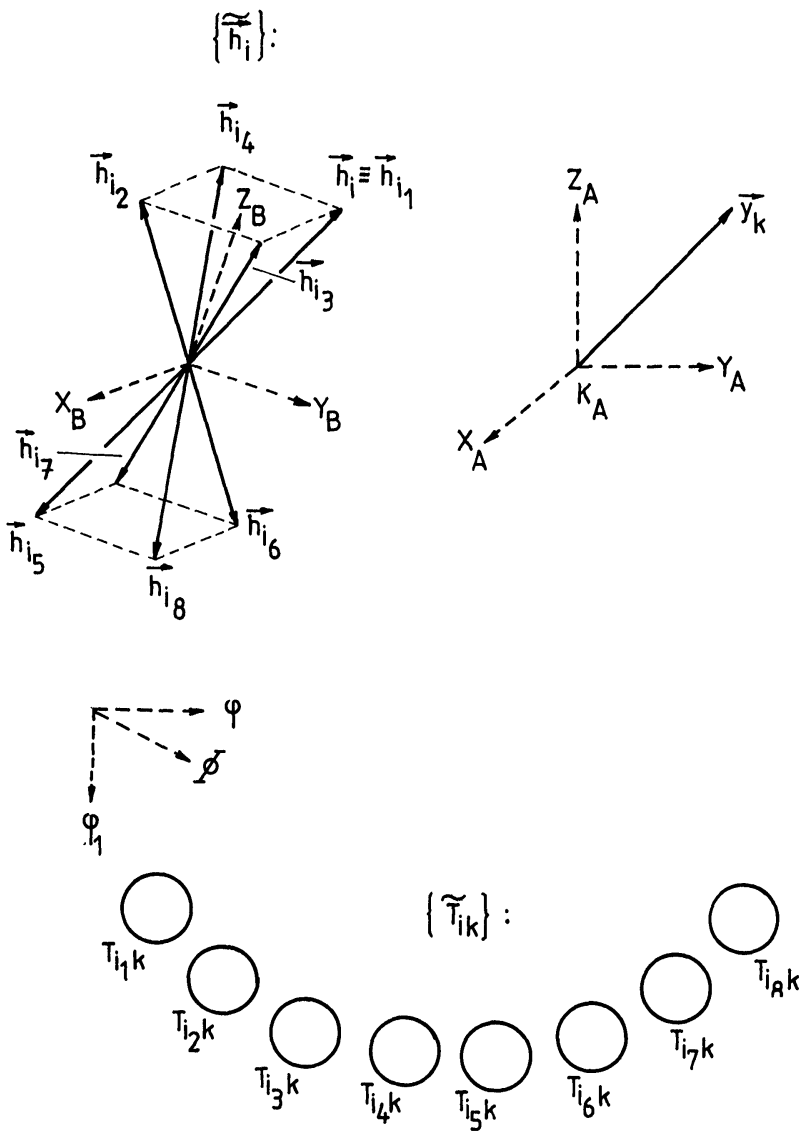

Figure 3 Example for the sets $\left\{\tilde{h}_{i}\right\}, T_{i_{\bar{e}} k}$ and $\left\{\tilde{T}_{i k}\right\}$ for the point symmetry group $\mathscr{G}_{B}=C_{2 v} . \vec{h}_{i}$ is an arbitrarily chosen crystal vector. By Eq. (14) we get $\tilde{N}_{b}=8$ vectors $\vec{h}_{i \cdot \vec{e}} \vec{y}_{k}$ is an arbitrary sample vector. For example, $T_{i_{5} k}$ contains all orientations in $G$ at which $\vec{h}_{i_{5}}$ is parallel to $\vec{y}_{k}$. The sum of the sets $T_{i_{\tilde{e}} k}(\tilde{e}=1, \ldots, 8)$ is denoted by $\left\{\tilde{T}_{i k}\right\}$.

\section{UNREDUCED POLE-FIGURES}

The distribution of crystallite orientations is described by the density function $f(g)$. All crystallites with orientation $g \in\left\{T_{i k}\right\}$ give one of their vectors $\vec{h}_{i_{e}}\left(e=1, \ldots, N_{b}\right)$ parallel to a fixed $\vec{y}_{k}$. The 
distribution of the vectors $\vec{h}_{i_{e}}$ in the polycrystalline sample is therefore described by the function

$$
P_{\left\{\vec{h}_{i}\right\}}\left(\vec{y}_{k}\right)=\frac{1}{\int_{\left\{T_{i k}\right\}} d g} \int_{\left\{T_{i k}\right\}} f(g) d g .
$$

$P_{\left\{\vec{h}_{i}\right\}}\left(\vec{y}_{k}\right)$ is called the unreduced pole-figure belonging to the crystal vector $\vec{h}_{i}$ and is in certain cases measurable by anomalous scattering.

\section{REDUCED POLE-FIGURES}

All crystallites with orientation $g \in\left\{\tilde{T}_{i k}\right\}$ give one of their vectors $\vec{h}_{i_{\tilde{e}}}\left(\tilde{e}=1, \ldots, \tilde{N}_{b}\right)$ parallel to a fixed $\vec{y}_{k}$. The distribution of the vectors $\vec{h}_{i_{\tilde{e}}}$ in the polycrystalline sample is described by the function

$$
P_{\left\{\tilde{h}_{i}\right\}}\left(\vec{y}_{k}\right)=\frac{1}{\int_{\left\{\tilde{T}_{i k}\right\}} d g} \int_{\left\{\tilde{T}_{i k}\right\}} f(g) d g .
$$

$P_{\left\{\tilde{h}_{i}\right\}}\left(\vec{y}_{k}\right)$ is called the reduced pole-figure belonging to the crystal vector $\vec{h}_{i}$ and can be measured by normal scattering.

\section{REPRODUCTION OF THE ORIENTATION DENSITY FUNCTION FROM POLE-FIGURES}

In Figure 4 the orientation of $K_{B}$ with respect to $K_{A}$ is $g$. The vectors $\vec{h}_{i_{e}}$ of set $\left\{\vec{h}_{i}\right\}$ are drawn in $K_{B}$ assuming that $G_{B}=C_{2 v}$. Let the sample vector $\vec{y}_{k_{s}}$ be parallel to the vectors $\vec{h}_{i_{e}}(e, s=$ $1, \ldots, N_{b}$ ). As we already know (see Figure 2) in all the cases when one of the vectors $\vec{h}_{i_{e}}$ is parallel to a fixed $\vec{y}_{k_{s}}$ the orientations of $K_{B}$ with respect to $K_{A}$ belong to the set $\left\{T_{i k_{s}}\right\}$. As the vectors $\vec{y}_{k_{s}}$ are chosen to be parallel to the vectors $\vec{h}_{i_{e}}$ of orientation $g$ every set $\left\{T_{i k_{s}}\right\}$ contains the orientation $g$, consequently, they are intersecting at $g$. If also different $i$ values are allowed $(i=1, \ldots, I)$ then the number of sets $\left\{T_{i k_{s}}\right\}$ intersecting at $g$ is $I \cdot N_{b}$. Note, that in the case of centrosymmetrical crystallites the sets $\left\{T_{i k_{s}}\right\}$ belonging to $\vec{y}_{k_{s}}$ and $-\vec{y}_{k_{s}}$ coincide.

After the Eq. (15) a value $P_{\left\{\vec{h}_{i}\right\}}\left(\vec{y}_{k_{s}}\right)$ gives information about the 


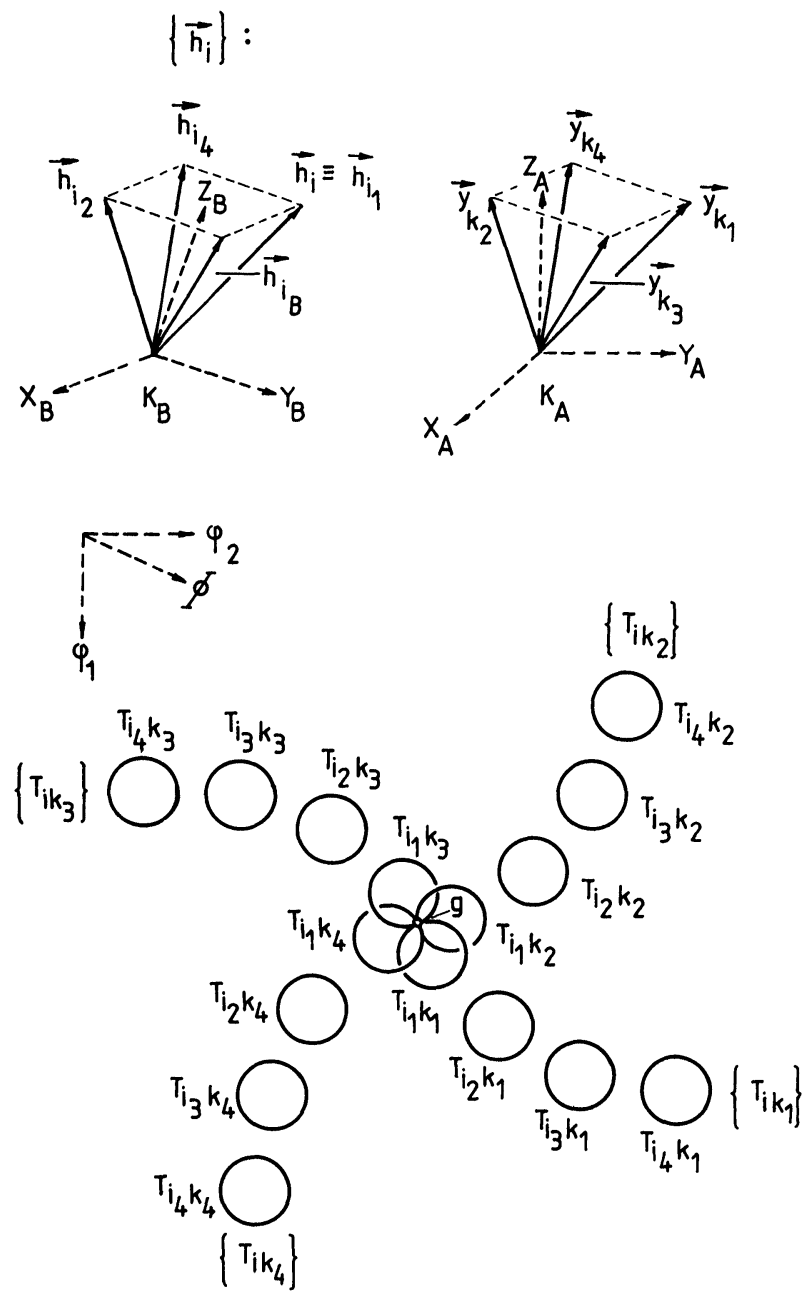

Figure 4 Schematic representation of $N_{b}$ intersecting sets $\left\{T_{i k}\right\}$. The orientation of $K_{B}$ with respect to $K_{A}$ is $g$. The set $\left\{\vec{h}_{i}\right\}$ is constructed in the same way as in Figure 2. The vectors of the set $\left\{\vec{y}_{k}\right\}=\left\{\vec{y}_{k_{1}}, \vec{y}_{k_{2}}, \ldots, \vec{y}_{k_{N_{b}}}\right\}$ are chosen to be parallel to the vectors $\vec{h}_{i_{1}}, \vec{h}_{i_{2}}, \ldots, \vec{h}_{i_{b}}$, respectively. All the sets $\left\{T_{i k_{s}}\right\}=$ $\left\{T_{i_{1} k_{s}}, T_{i_{2} k_{s}}, \ldots, T_{i_{N_{b}} k_{s}}\right\},\left(s=1,2, \ldots, N_{b}\right)$ are containing the orientation $g$ and, consequently, they intersect at $g$. 
orientation density function in the set $\left\{T_{i k_{s}}\right\}$. To the common point $g$ at which the sets $\left\{T_{i k_{s}}\right\}$ intersect belong $I \cdot N_{b}$ values $P_{\left\{\vec{h}_{i}\right\}}\left(\vec{y}_{k_{s}}\right)$ informing us about the common value $f(g)$ at the same time. We couple the unknown $f(g)$ value with its information by multiplying them. From Eq. (15) we get

$$
f(g) \cdot P_{\left\{\vec{h}_{i}\right\}}\left(\vec{y}_{k_{s}}\right)=f(g) \frac{1}{\int_{\left\{T_{i_{s}}\right\}} d g} \int_{\left\{T_{\left.i_{s}\right\}}\right\}} f(g) d g, \begin{aligned}
& i=1, \ldots, I \\
& s=1, \ldots, N_{b}
\end{aligned} .
$$

After rearranging we get

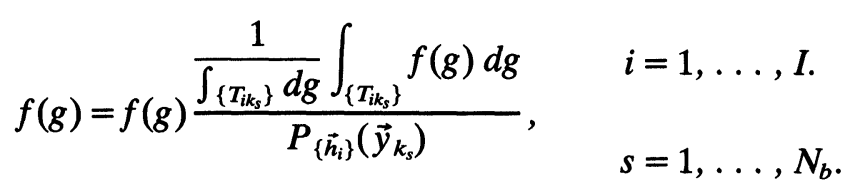

or

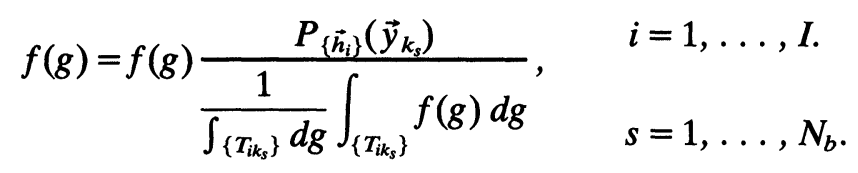

Let us perform the operation prescribed by the right side of Eq. (17a) with an approaching function $f^{(N)}(g)$. The numerator gives an approaching pole-figure value denoted by $P_{\left\{\vec{h}_{i}\right\}}^{(N)}\left(\vec{y}_{k_{s}}\right)$. In the denominator is the measured pole-figure data. The multiplication of $f^{(N)}(g)$ with the ratio $P_{\left\{\vec{h}_{i}\right\}}^{(N)}\left(\vec{y}_{k_{s}}\right) / P_{\left\{h_{i}\right\}}\left(\vec{y}_{k_{s}}\right)$ results in the $(n+1)$ th approximation of $f(g)$, i.e.:

$$
f^{(N+1)}(g)=f^{(N)}(g) \frac{P_{\left\{\vec{h}_{i}\right\}}^{(N)}\left(\vec{y}_{k_{s}}\right)}{P_{\left\{\vec{h}_{i}\right\}}\left(\vec{y}_{k_{s}}\right)}, \quad \begin{aligned}
& i=1, \ldots, I . \\
& s=1, \ldots, N_{b} .
\end{aligned}
$$

Quite analogously from Eq. (17b) follows

$$
f^{(N+1)}(g)=f^{(N)}(g) \frac{P_{\left\{\vec{h}_{i}\right\}}\left(\vec{y}_{k_{s}}\right)}{P_{\left\{\vec{h}_{i}\right\}}^{(N)}\left(\vec{y}_{k_{s}}\right)}, \quad \begin{aligned}
& i=1, \ldots, I . \\
& s=1, \ldots, N_{b} .
\end{aligned}
$$

$P_{\left\{\vec{h}_{i}\right\}}^{(N)}\left(\vec{y}_{k_{s}}\right)$ means the unreduced pole-figure recalculated from the approaching function $f^{(N)}(g)$. After taking the average of Eqs (19) we get a formula for approximative determination of $f(g)$ contain- 
ing all pole-figure data allocated to the orientation $g$.

$$
f^{(N+1)}(g)=f^{(N)}(g) \cdot \underset{\substack{i=1, \ldots, I \\ s=1, \ldots, N_{b}}}{\text { Average }}\left\{\frac{P_{\left\{\vec{h}_{i}\right\}}\left(\vec{y}_{k_{s}}\right)}{P_{\left\{\vec{h}_{i}\right\}}^{(N)}\left(\vec{y}_{k_{s}}\right)}\right\} .
$$

A possible average is for example the following (Imhof, 1983a, 1983b):

$$
f^{(N+1)}(g)=f^{(N)}(g)\left[\frac{1}{I \cdot N_{b}} \sum_{i=1}^{I} \sum_{s=1}^{N_{b}}\left(\frac{P_{\left\{\vec{h}_{i}\right\}}\left(\vec{y}_{k_{s}}\right)}{P_{\left\{\vec{h}_{i}\right\}}^{(N)}\left(\vec{y}_{k_{s}}\right)}\right)^{E}\right]^{1 / E} .
$$

$E$ is a real number. With $E=1$ we get the algebraical average of the Eqs (19), while $E=-1$ gives the harmonic average of them.

In the same way can be obtained the corresponding general formula of the iterative reproduction method using reduced polefigures:

$$
f^{(N+1)}(g)=f^{(N)}(g) \cdot \underset{\substack{i=1, \ldots, I \\ \tilde{s}=1, \ldots, N_{b}}}{\text { Average }}\left\{\frac{P_{\left\{\tilde{h}_{i}\right\}}\left(\vec{y}_{k_{s}}\right)}{P_{\left\{\tilde{h}_{i}\right\}}^{(N)}\left(\vec{y}_{k_{s}}\right)}\right\} .
$$

To Eq. (21) corresponds then

$$
f^{(N+1)}(g)=f^{(N)}(g)\left[\frac{1}{I \cdot \tilde{N}_{b}} \sum_{i=1}^{I} \sum_{\tilde{s}=1}^{\tilde{N}_{b}}\left(\frac{P_{\left\{\tilde{h}_{i}\right\}}\left(\vec{y}_{k_{s}}\right)}{P_{\left\{\tilde{h}_{i}\right\}}^{(N)}\left(\vec{y}_{k_{s}}\right)}\right)^{E}\right]^{1 / E} .
$$

Here the sets $\left\{\tilde{T}_{i k_{\tilde{s}}}\right\}$ belonging to $\vec{y}_{k_{\tilde{s}}}$ and $-\vec{y}_{k_{s}}$ coincide in all cases. If the sample consists of centrosymmetrical crystallites then $\tilde{N}_{b}=$ $N_{b},\left\{\vec{h}_{i}\right\}=\left\{\vec{h}_{i}\right\}$ and $\left\{\tilde{T}_{i k_{s}}\right\}=\left\{T_{i k_{s}}\right\}$. In other cases the integration sets $\left\{\tilde{T}_{i k_{s}}\right\}$ are larger than the corresponding sets $\left\{T_{i k_{s}}\right\}$ and the reduced pole-figure data are therefore less informative about $f(g)$ than the unreduced ones.

\section{INITIAL FUNCTION OF THE ITERATION}

The most simple form of Eqs (20) and (22) can be got by choosing an initial function $f^{(1)}(g)$ resulting in isotropic pole-figures. In this 
case Eqs (20) and (22) turn into

$$
\begin{gathered}
f^{(2)}(g)=f^{(1)}(g) \cdot \underset{\substack{i=1, \ldots, I \\
s=1, \ldots, N_{b}}}{\text { Average }}\left\{P_{\left\{\vec{h}_{i}\right\}}\left(\vec{y}_{k_{s}}\right)\right\} \\
f^{(2)}(g)=f^{(1)}(g) \cdot \underset{\substack{i=1, \ldots, I \\
\tilde{s}=1, \ldots, N_{b}}}{\text { Average }}\left\{P_{\left\{\vec{h}_{i}\right\}}\left(\vec{y}_{k_{s}}\right)\right\}
\end{gathered}
$$

respectively. After Bunge and Esling (1979) an infinite number of functions $f^{(1)}(g)$ exists resulting in isotropic reduced pole-figures $P_{\left\{\vec{h}_{i}\right\}}^{(1)}\left(\vec{y}_{k_{s}}\right) \equiv 1$. If the measured reduced pole-figures are also isotropic ones then $f^{(N)}(g)=f^{(2)}(g)=f^{(1)}(g)$, i.e., depending on the choice of $f^{(1)}(g)$ we get an infinite number of solutions. We need therefore to decide for one of them. The reason of the choice of the function $f^{(1)}(g) \equiv 1$ from among the other possible solutions is as follows. At the very beginning of the iteration we do not know anything about the function $f(g)$ and therefore we must give the same chance for any orientation to take part in the reproduced $f(g)$. With $f^{(1)}(g) \not \equiv 1$ it is not the case. The choice of $f^{(1)}(g) \not \equiv 1$ supposes an additional method which leads to the concrete form of $f^{(1)}(g)$. But this is in contradiction with the fact that at the beginning we do not know anything about the $f(g)$. With $f^{(1)}(g) \equiv 1$ we get

$$
\begin{gathered}
f^{(2)}(g)=\underset{\substack{i=1, \ldots, I \\
s=1, \ldots, N_{b}}}{\text { Average }}\left\{P_{\left\{\vec{h}_{i}\right\}}\left(\vec{y}_{k_{s}}\right)\right\} \\
f^{(2)}(g)=\underset{\substack{i=1, \ldots, I \\
s=1, \ldots, \hat{N}_{b}}}{\text { Average }}\left\{P_{\left\{\tilde{h}_{i}\right\}}\left(\vec{y}_{k_{s}}\right)\right\} .
\end{gathered}
$$

For example, the geometric average in Eq. (27) gives the function

$$
f_{W}^{(2)}(g)=\prod_{i=1}^{I} \prod_{s=1}^{\tilde{N}_{b}}\left(P_{\left\{\tilde{h}_{i}\right\}}\left(\vec{y}_{k_{5}}\right)\right)^{1 /\left(I \cdot \tilde{N}_{b}\right)}
$$

Matthies and Vinel (1982) use this function as an initial function in the procedure (22):

$$
f_{W I M V}^{(1)}(g)=f_{W}^{(2)}(g) \text {. }
$$

The WIMV-method is therefore based last of all on the choice of $f^{(1)}(g) \equiv 1$, too. A product-function analogous to $(28)$ was first used by Williams (1968) in the texture analysis. 


\section{CONTINUOUS REPRESENTATION}

According to the Bunge-Roe formalism (Matthies, 1982) both the orientation density function and the pole-figures can be developed into series of spherical functions $D_{m n}^{l}(g), Y_{l m}(\vec{y})$ :

$$
\begin{gathered}
f(g)=\sum_{l=0(1)}^{\infty} \sum_{m, n=-l}^{l} C_{l}^{m n} D_{m n}^{l}\left(g^{-1}\right) \\
P_{\left\{\tilde{h}_{i}\right\}}(\vec{y})=\frac{1}{\tilde{N}_{b}} \sum_{\vec{e}=1}^{\tilde{N}_{b}} \sum_{l=0(1)}^{\infty} \sum_{m, n=-l}^{l} \frac{4 \pi}{2 l+1} C_{l}^{m n} Y_{l m}^{*}\left(\vec{h}_{i_{\vec{e}}}\right) Y_{l n}(\vec{y}) .
\end{gathered}
$$

With the assignation

$$
F_{l}^{n}\left(\left\{\vec{h}_{i}\right\}\right)=\frac{1}{\tilde{N}_{b}} \sum_{\vec{e}=1}^{\tilde{N}_{b}} \sum_{m=-l}^{l} C_{l}^{m n} Y_{l m}^{*}\left(\vec{h}_{i_{\bar{e}}}\right)
$$

we get

$$
P_{\left\{\tilde{\hat{h}_{i}}\right\}}(\vec{y})=\sum_{l=0(1)}^{\infty} \sum_{n=-l}^{l} F_{l}^{n}\left(\left\{\tilde{h}_{i}\right\}\right) Y_{l n}(\vec{y})
$$

The inverse relations are

$$
\begin{aligned}
& C_{l}^{m n}=\frac{2 l+1}{8 \pi^{2}} \int_{G} f(g) D_{n m}^{l}(g) d g \\
& F_{l}^{n}\left(\left\{\tilde{h}_{i}\right\}\right)=\int_{Y} P_{\left\{\tilde{h}_{i}\right\}}(\vec{y}) Y_{l n}^{*}(\vec{y}) d \vec{y} .
\end{aligned}
$$

$G$ is the domain given by the relations (5) and $Y$ means the space of sample vectors $\vec{y}$.

The pole-figure data are generally given at discrete vectors $\vec{y}$ and so the values $F_{l}^{n}\left(\left\{\hat{h}_{i}\right\}\right)$ can be calculated from (35) by numerical integration. Equation (32) is still not suitable for determination of all the coefficients $C_{l}^{m n}$ because the $l$-odd coefficients are covered by a factor $\left(1+(-1)^{l}\right)$ (Matthies, 1982).

The pole-figures are functions obtained from the distribution of orientations. Equation (22) allows us to follow the same way: from an approaching $f^{(N)}(g)$ to recalculate the $N$ th approximation of the pole figures together being the blocks of the next approach $f^{(N+1)}(g)$.

First we construct the set $\left\{\tilde{\hat{h}_{i}}\right\}$ in the coordinate system $K_{B}$ which 
is oriented with respect to $K_{A}$ by $g$. The vectors $\vec{y}_{k_{s}}\left(\tilde{s}=1,{ }_{\tilde{z}} ., \tilde{N}_{b}\right)$ in $K_{A}$ are chosen to be parallel to the vectors of the set $\left\{\tilde{h}_{i}\right\}$. The pole-figure values $P_{\left\{\tilde{h}_{i}\right\}}\left(\vec{y}_{k_{s}}\right)$ in the numerator of (22) are to be calculated by the Eq. (33).

We start the iteration with $f^{(1)}(g) \equiv 1$ and by Eq. (27) we get $f^{(2)}(g)$, of course, at discrete orientations we have chosen. The corresponding coefficients ${ }^{(2)} C_{l}^{m n}$ can be calculated from Eq. (34) and the approaching pole-figure values $P_{\left\{h_{i}\right\}}^{(2)}\left(\vec{y}_{k_{s}}\right)$ from (31) using the $l$-even $C_{l}^{m n}$ only. Substituting the recalculated values $P_{\left\{h_{i}\right\}}^{(2)}\left(\vec{y}_{k_{s}}\right)$ into the denominator of (22), with the known $f^{(2)}(g)$ we get $f^{(3)}(g)$ at the same discrete orientations. By this procedure we get a solution $f^{(N)}(g)$ with coefficients $C_{l}^{m n}$ coupled to the assumption $f^{(1)}(g) \equiv 1$ and to the concrete averaging operator.

All the corresponding expressions connected with unreduced pole-figures can be got by substituting $\left\{\vec{h}_{i}\right\}$ and $N_{b}$ instead of $\left\{\vec{h}_{i}\right\}$ and $\tilde{N}_{b}$, respectively. If the sample consists of centro-symmetrical crystallites the recalculation of the values $P_{\left\{\vec{h}_{i}\right\}}^{(N)}\left(\vec{y}_{k_{s}}\right)$ can also be done with $l$-even $C_{l}^{m n}$ only.

\section{DISCRETE REPRESENTATIONS}

A. For practical reasons the vectors inside the regions $\Delta Y_{k}$ cannot be distinguished. $\Delta Y_{k}$ denotes the $k$ th subset of the space $Y$. The size of $\Delta Y_{k}$ is determined by the measuring equipment and represents the resolving power of the pole-figures. The function $P_{\left\{\tilde{h}_{i}\right\}}(\vec{y})$ is constant inside $\Delta Y_{k}$ and is denoted by $P_{\left\{\tilde{h}_{i}\right\}}\left(\Delta Y_{k}\right)$. The set $\left\{\tilde{T}_{i k}\right\}$ contains now all possible orientations in the domain $G$ at which one of the vectors $\vec{h}_{i_{\bar{e}}}$ point into the region $\Delta Y_{k}$.

Let an orientation give its vectors $\vec{h}_{i_{\tilde{e}}}$ into the regions $\Delta Y_{k_{\tilde{s}}}(\tilde{e}, \tilde{s}=$ $\left.1, \ldots, \tilde{N}_{b}\right)$. All those orientations which give one of their vectors $\vec{h}_{i_{\bar{e}}}$ into the same regions $\Delta Y_{k_{\tilde{s}}}$ are classified into an orientation class $C_{j}(j=1, \ldots, \mathscr{F})$. Orientations $g \in C_{j}$ cannot be distinguished on the base of the resolving power of pole-figures. The function $f(g)$ is constant inside one class and is denoted by $f\left(C_{j}\right)$. For any orientation $g \in C_{j}$ Eq. (22) reads then

$$
f^{(N+1)}\left(C_{j}\right)=f^{(N)}\left(C_{j}\right) \cdot \underset{\substack{i=1, \ldots, I \\ \tilde{s}=1, \ldots, \bar{N}_{b}}}{\text { Average }}\left\{\frac{P_{\left\{\tilde{h}_{i}\right\}}\left(\Delta Y_{k_{s}}\right)}{P_{\left\{\bar{n}_{i}\right\}}^{(N)}\left(\Delta Y_{k_{s}}\right)}\right\} .
$$


To the concrete average (23) corresponds

$$
f^{(N+1)}\left(C_{j}\right)=f^{(N)}\left(C_{j}\right)\left[\frac{1}{I \cdot \tilde{N}_{b}} \sum_{i=1}^{I} \sum_{\tilde{s}=1}^{\tilde{N}_{b}}\left(\frac{P_{\left\{\tilde{h}_{i}\right\}}\left(\Delta Y_{k_{s}}\right)}{P_{\left\{\tilde{h}_{i}\right\}}^{(N)}\left(\Delta Y_{k_{\bar{s}}}\right)}\right)^{E}\right]^{1 / E} .
$$

The expressions corresponding to unreduced pole-figures (Imhof, 1983a, 1983b) can be got by writing $\left\{\vec{h}_{i}\right\}$ and $N_{b}$ instead of $\left\{\vec{h}_{i}\right\}$ and $\tilde{N}_{b}$, respectively.

B. Applying the definitional Eq. (6) to the classes $C_{j}$ we get

$$
\frac{\Delta V}{V}\left(g \in C_{j}\right)=f\left(C_{j}\right) \frac{V\left(C_{j}\right)}{V(G)} .
$$

$V(G)$ : volume of the domain $G, V\left(C_{j}\right)$ : volume of the subset $C_{j}$.

With fixed $\Delta \varphi_{1}, \Delta \phi, \Delta \varphi_{2}$ we define discrete points in the Eulerian space by the expression $n_{1} \Delta \varphi_{1}+n_{2} \Delta \phi+n_{3} \Delta \varphi_{2}$, where $n_{1}, n_{2}$ and $n_{3}$ are integer numbers. The bisecting planes between the discrete points define parallelepipeds around them. Let $C_{j_{1}}$ contain the point $\left(n_{1}, n_{2}, n_{3}\right)$ and $M$ the number of those classes $C_{j m}(m=$ $1, \ldots, M)$, the volume of which is entirely or for the most part in the parallelepiped around the point $\left(n_{1}, n_{2}, n_{3}\right)$. The union $C E=$ $C_{j_{1}} \cup C_{j_{2}} \ldots \cup C_{j_{M}}$ is called the cell. In this way the Eulerian space is divided into cells filling in the region $G$ and excluding each other.

The volume fraction of those crystallites the orientation of which

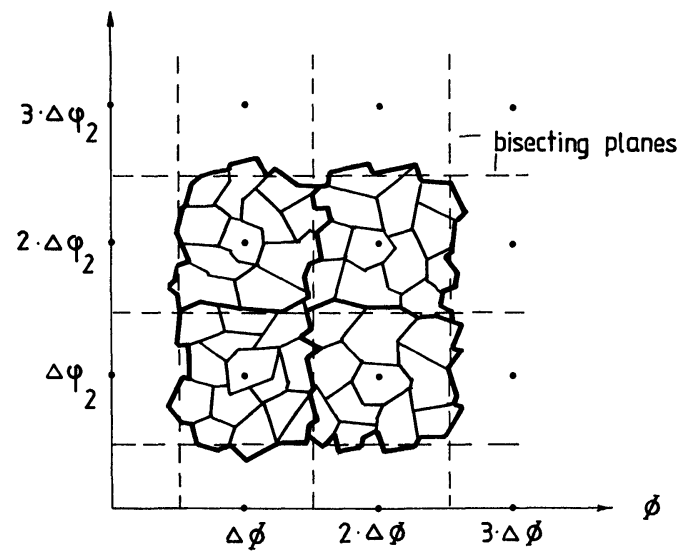

Figure 5 Schematic representation of the cells in two dimensions. - boundary of classes; — boundary of cells; $\cdot$ discrete points defined by $n_{2} \Delta \phi+n_{3} \Delta \varphi_{2}$. 
is in the cell $C E$ comes from (38) as follows:

$$
\begin{aligned}
\frac{\Delta V}{V}(g \in C E)=\sum_{m=1}^{M} & \frac{\Delta V}{V}\left(g \in C_{j_{m}}\right) \\
& =\left(\sum_{m=1}^{M} f\left(C_{j_{m}}\right) \frac{V\left(C_{j_{m}}\right)}{V(C E)}\right) \frac{V(C E)}{V(G)} .
\end{aligned}
$$

On the analogy of Eq. (38) one can define the mean value of the orientation density function in the cell $C E$ (Imhof, 1985)

$$
f(C E)=\sum_{m=1}^{M} f\left(C_{j_{m}}\right) \frac{V\left(C_{j m}\right)}{V(C E)} .
$$

Let us substitute $f^{(N+1)}\left(C_{j m}\right)$ (Eq. 36) for the $f\left(C_{j m}\right)$. Inside the cell the orientation density function is assumed to be constant, i.e., $f^{(N)}\left(C_{j m}\right)=f^{(N)}(C E)$. After summarizing over $m$ by constant $i$ and $\tilde{s}$ we get

$$
f^{(N+1)}(C E)=f^{(N)} \cdot \underset{\substack{i=1, \ldots, I \\ s=1, \ldots, N_{b} \\ m^{\prime}=1, \ldots, M^{\prime}}}{\text { Average }}\left\{\frac{P_{\left\{\tilde{h}_{i}\right\}}\left(\Delta Y_{k_{s}}\right)_{m^{\prime}}}{P_{\left\{\tilde{h}_{i}\right\}}\left(\Delta Y_{k_{s}}\right)_{m^{\prime}}} \cdot \frac{V\left(\left\{T_{i k_{s}}\right\}_{m^{\prime}} \cap C E\right.}{V(C E)}\right\} .
$$

$V\left(\left\{T_{i k_{s}}\right\}_{m^{\prime}} \cap C E\right)$ : volume of section of the set $\left\{T_{i k_{s}}\right\}_{m^{\prime}}$ with the cell $C E$.

For constant indices $i$ and $\tilde{e}$ the vectors $\vec{h}_{i_{\tilde{e}}}$ of different orientations $g \in C_{j}$ point always into the same $\Delta Y_{k_{s}}$; in the case of the orientations $g \in C E$, however, they point into more than one $\Delta Y_{k_{s}}$, numbered by $m^{\prime}$. The set $\left\{T_{i k_{s}}\right\}$ corresponding to the region $\left(\Delta Y_{k_{5}}\right)_{m^{\prime}}$ is denoted by $\left\{T_{i k_{5}}\right\}_{m^{\prime}}$.

The quantities in (41) refer to the whole cell $C E$ and do already not depend on the form of the cell. Thus, for example, for an arbitrarily chosen cell the Eq. (37) modifies to

$$
\begin{aligned}
& f^{(N+1)}(C E)=f^{(N)}(C E)\left[\frac{1}{I \cdot \tilde{N}_{b}} \sum_{i=1}^{I} \sum_{i=1}^{\tilde{N}_{b}} \sum_{m^{\prime}=1}^{M^{\prime}}\right. \\
& \left.\left(\frac{P_{\left\{\tilde{h}_{i}\right\}}\left(\Delta Y_{k_{s}}\right)_{m^{\prime}}}{P_{\left\{\bar{h}_{i}\right\}}^{(N)}\left(\Delta Y_{k_{\bar{s}}}\right)_{m^{\prime}}} \cdot \frac{V\left(\left\{T_{i k_{s}}\right\}_{m^{\prime}} \cap C E\right)}{V(C E)}\right)^{E}\right]^{1 / E} .
\end{aligned}
$$

The expressions corresponding to the unreduced pole-figures can be 
got by writing $\left\{\vec{h}_{i}\right\}$ and $N_{b}$ instead of $\left\{\vec{h}_{i}\right\}$ and $\tilde{N}_{b}$, respectively. Equations (41) and (42) were derived also by Pawlik (1986) on the base of probability quantities.

In the vector-method (Ruer and Baro, 1977) the cells $C E_{j}(j=$ $1, \ldots, \mathscr{F})$ are of form of parallelepiped. The value $f\left(C E_{j}\right)$ is considered to be the $j$ th component of the column vector $\vec{F}$. Each pole-figure is divided into regions $\Delta Y_{k},(k=1, \ldots, K)$. The polefigure data $P_{\left\{\tilde{h}_{i}\right\}}\left(\Delta Y_{k}\right)$ measured at the region $\Delta Y_{k}$ is considered to be the $k$ th component of the column vector $\vec{I}\left(\left\{\vec{h}_{i}\right\}\right)$. We denote the set of all the orientations of the domain $G$ giving one of their vectors $\vec{h}_{i \bar{e}}$ into the region $\Delta Y_{k}$ henceforward by $\left\{\tilde{T}_{i k}\right\}$. The volume of the set $\left\{\tilde{T}_{i k}\right\}$ is denoted by $V\left(\left\{\tilde{T}_{i k}\right\}\right)$ and the volume of the section $\left\{\tilde{T}_{i k}\right\} \cap C E_{j}$ by $V\left(\left\{\tilde{T}_{i k}\right\} \cap C E_{j}\right)$. For the measured polefigure data we can write the following equation corresponding to Eq. (16)

$$
P_{\left\{\tilde{h}_{i}\right\}}\left(\Delta Y_{k}\right)=\frac{1}{V\left(\left\{\tilde{T}_{i k}\right\}\right)} \sum_{j=1}^{\mathscr{F}} V\left(\left\{\tilde{T}_{i k}\right\} \cap C E_{j}\right) \cdot f\left(C E_{j}\right) .
$$

As the set $\left\{\tilde{T}_{i k}\right\}$ intersect a restricted number of cells only, a great number of members under the summation equals to zero. The summation is still made over all the possible values of $j$ because it allows us to write the system of Eqs $(43)(k=1, \ldots, K)$ in the form

$$
\vec{I}\left(\left\{\vec{h}_{i}\right\}\right)=\left[\sigma_{k j}\left(\left\{\tilde{\hat{h}_{i}}\right\}\right)\right] \cdot \vec{F}
$$

$\left[\sigma_{k j}\left(\left\{\hat{h}_{i}\right\}\right)\right]=a K \times \mathscr{F}$ matrix with $\mathscr{F}$ columns and $K$ arrows and

$$
\sigma_{k j}=\frac{V\left(\left\{\tilde{T}_{i k}\right\} \cap C E_{j}\right)}{V\left(\left\{\tilde{T}_{i k}\right\}\right)} .
$$

The volumes of the sections $\left\{\tilde{T}_{i k_{s}}\right\}_{m^{\prime}} \cap C E$ which appear in Eq. (41) are in the numerators of the non-zero elements of a column of the matrix $\left[\sigma_{k j}\left(\left\{\hat{h}_{i}\right\}\right)\right]$. The columns itself are called by Ruer and Baro the elementary pole-figures. This is the relation between the discrete representation (41) of the iteration method (22) and the vector-method.

C. Let the cells be chosen to be parallelepipeds around the points $\left(n_{1}, n_{2}, n_{3}\right)$ and let in the Eq. (27) $g$ mean the point $\left(n_{1}, n_{2}, n_{3}\right)$. Then the sample vectors $\vec{y}_{k_{s}}$ are parallel to the crystal vectors $\vec{h}_{i_{\tilde{e}}}\left(\tilde{e}, \tilde{s}=1, \ldots, \tilde{N}_{b}\right)$ of $g$. The recalculation of the values $P_{\left\{\tilde{h}_{i}\right\}}^{(N)}\left(\vec{y}_{\tilde{s}}\right)$ 
occurs by the Eq. (16) with the difference that $f(g)$ inside one cell is considered to be constant. This way is followed by Matthies and Vinel (1982) in the WIMV-method.

\section{SUMMARY}

A reproduction method was obtained valid for single fase polycrystalline sample consisting of crystallites with any point symmetry group. In the case of enantiomorphic crystallites only one form of them is assumed to be present in the sample.

The kernel of the method is the Eq. (20) and the one (22). After applying them to the points and to subsets of orientation space we get different representations of the same method. The continuous representation must give the most accurate results because here is not assumed that the orientation density function is constant inside the subsets of the orientation space.

The reproducibility of the orientation density function is directly connected with the length of the integration paths $\left\{T_{i k}\right\}$ or $\left\{\tilde{T}_{i k}\right\}$. Generally some of the subsets $T_{i_{e} k}$ or $T_{i_{i} k}$ are equivalent. The larger is the number of non-equivalent subsets in the union $\left\{T_{i k}\right\}$ or $\left\{\tilde{T}_{i k}\right\}$ the less informative are the pole-figure data about the orientation density function.

\section{References}

Bradley, C. J. and Cracknell, A. P. (1972). The Mathematical Theory of Symmetry in Solids, Clarendon Press, Oxford.

Bunge, H. J. (1969). Mathematische Methoden der Texturanalyse. Akademie-Verlag, Berlin.

Bunge, H. J. and Esling, C. (1979). Texture 3, 169.

Bunge, H. J., Esling, C. and Müller, J. (1982). Recent Developments in Texture Analysis, in Quantitative Texture Analysis, Edited by Bunge, H. J. and Esling, C., DGM, Oberursel, 477.

Imhof, J. (1983a). Phys. Stat. Sol (b) 119, 693.

Imhof, J. (1983b). Phys. Stat. Sol (b) 120, 321.

Imhof, J. (1985). Collected Abstracts of the XIIth Hungarian Diffraction Conference. Sopron, 93.

Matthies, S. (1982). Aktuelle Probleme der quantitativen Texturanalyse. Rossendorf.

Matthies, S. and Vinel, G. W. (1982). Phys. Stat. Sol (b) 112, K111 and K115.

Pawlik, K. (1986). Phys. Stat. Sol (b) 134, 477.

Pospiech, J. (1980). Kristall und Technik, 15, 1481.

Ruer, D. and Baro, R. (1977). Adv. X-Ray Analysis, 20, 187.

Williams, R. O. (1968). J. appl. Phys. 32, 4329. 